\title{
Estandarización del proceso de confección, a través de la ingeniería de métodos, para aumentar la productividad, en una empresa del ramo textil en el estado de Puebla
}

\section{Standardization of the making process, through method engineering, to increase productivity, in a company in the textile industry in the state of Puebla}

\author{
VELÁZQUEZ-MANCILLA, Jorge Enrique†*, FIERRO-XOCHITOTOTL, María Concepción y \\ CHÁVEZ-MEDINA, Juan
}

Instituto Tecnológico Superior de San Martín Texmelucan, México.

ID $1^{\mathrm{er}}$ Autor: Jorge Enrique, Velázquez-Mancilla / ORC ID: 0000-0002-0176-1923, Researcher ID Thomson: X-22282018, CVU CONACYT ID: 510054

ID $1{ }^{\text {er }}$ Coautor: María Concepción, Fierro-Xochitototl / ORC ID: 0000-0001-6967-0131, Researcher ID Thomson: X-22092018

ID $2^{\text {do }}$ Coautor: Juan, Chávez-Medina / ORC ID: 0000-0002-1978-0620, CVU CONACYT ID: 417889

DOI: $10.35429 / J I E .2020 .13 .4 .1 .7$

Recibido Julio 10, 2020; Aceptado Diciembre 30, 2020

Resumen

La estandarización de un proceso permite validar un método para alcanzar los objetivos de producción y las características de calidad requeridas, sin embargo, aún existen empresas que hoy en día, permiten que cada trabajador desarrolle su propio método, lo cual repercute negativamente en el logro de esos objetivos. Cuando se presenta un problema de calidad, se vuelve complejo encontrar la causa raíz, ya que derivará de diferentes métodos de trabajo. En este sentido, el proceso de capacitación falla, al no establecer específicamente el proceso correcto a seguir para cada operación. Estas fallas repercuten tanto en los ingresos como en las ganancias que puedan tener las empresas, es por ello la relevancia de contar con estándares de trabajo. El objetivo del presente trabajo es determinar el estándar del proceso de confección, a través de la Ingeniería de Métodos, para aumentar la productividad en una empresa del ramo textil, dedicada a la fabricación de ropa deportiva. El resultado de esta investigación muestra un resultado positivo, entre el establecimiento de un estándar y el aumento de la productividad.

Estandarización, Ingeniería de métodos, Proceso de confección, Industria textil

\begin{abstract}
The standardization of a process allows to validate a method to achieve the production objectives and the required quality characteristics, however, there are still companies that today allow each worker to develop their own method, which negatively affects the achievement of those goals. When a quality problem arises, it becomes complex to find the root cause, since it will derive from different working methods. In this context, the training process fails, by not specifically establishing the correct process to follow for each operation. These failures affect both the income and the profits that the companies may have, which is why it is relevant to have work standards. The objective of this work is to determine the standard of the making process, through Method Engineering, to increase productivity in a company in the textile industry, dedicated to the manufacture of sportswear. The result of this research shows a positive result, between setting a standard and increasing productivity.
\end{abstract}

Standardization, Method Engineering, making process, textile industry

Citación: VELÁZQUEZ-MANCILLA, Jorge Enrique, FIERRO-XOCHITOTOTL, María Concepción y CHÁVEZMEDINA, Juan. Estandarización del proceso de confección, a través de la ingeniería de métodos, para aumentar la productividad, en una empresa del ramo textil en el estado de Puebla. Revista de Ingeniería Industrial. 2020. 4-13:1-7.

\footnotetext{
* Correspondencia del Autor (Email: vmancilla_enrique@ hotmail.com)

$\uparrow$ Investigador contribuyendo como primer autor.
} 


\section{Introducción}

Aproximadamente 120 millones de habitantes conforman la República Mexicana, de los cuales la población joven entre 12 y 29 años representa el $31.4 \%$; esto es relevante dado que los jóvenes son el mercado más importante para el sector moda y textil en México (CEMDA, 2019).

En específico la industria textil se compone de alrededor de 20,000 empresas, las cuales contribuyen con el $10 \%$ del PIB en el sector manufacturero (Gobierno Fácil, 2020). El 90\% de las empresas del ramo textil son PyMEs, y generan cerca de un millón de empleos tanto directos como indirectos (Gobierno Fácil, 2020). El Estado de Puebla es la novena economía del país, siendo sus mayores sectores el de la industria automotriz y el de la manufactura, que considera la maquila, en especial los productos textiles (Secretaría de Economía, 2019).

Existen diferentes trabajos que muestran la relación positiva del uso de la ingeniería de métodos para mejorar la rentabilidad de la organización (Carbanillas y León Ríos, 2020; Bazan, 2019; Castillo, 2019; Ruiz, 2016; Ramírez, 2015).

El presente artículo tiene por objetivo demostrar la importancia que tiene el establecimiento de un método estandarizado para mejorar la productividad y la calidad de las áreas productivas.

\section{Situación actual de la empresa}

La empresa caso de estudio se encuentra ubicada en el Estado de Puebla, pertenece al ramo textil, tiene cerca de una década en el mercado y es clasificada como mediana; dedicada a la producción de ropa deportiva y ropa interior para la familia. Reconocida por su incursión a nivel internacional, en la exportación de prendas pertenecientes a importantes marcas dentro del sector.

Debido a la naturaleza del proceso productivo y teniendo en cuenta que hay un constante cambio de diseños y estilos que posiblemente nunca se volverán a reprogramar o que no son muy constantes, ha sido difícil para la empresa establecer tiempos fijos para cada proceso.
Dentro de las necesidades que la empresa presenta, están las altas demandas de producto y estilos de prendas, y el hecho que no se llegan a cumplir las metas satisfactoriamente ocasionando una baja eficiencia en el área productiva, aunado a que en la mayoría de las ocasiones los operadores no suelen ser aprovechados al $100 \%$ para cubrir estas tareas. Generalmente los operadores realizan actividades dentro del proceso sobre las cuales no tienen un conocimiento previo, generando problemas por índices altos de retrabajos.

De manera general, el proceso de producción integra los siguientes pasos: tejido, teñido y secado, confección, inspección, prehormado, hormado, acabado y verificado.

El porcentaje semanal promedio de cumplimiento a las metas de producción, que se ha tenido es el que se muestra en el gráfico 1. Estos valores fueron obtenidos de los registros de producción que se tienen en la empresa caso de estudio.

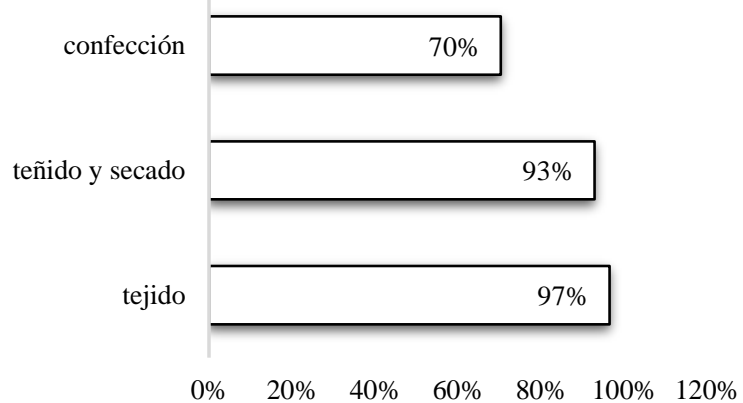

Gráfico 1 Porcentaje de cumplimiento de producción Fuente: Elaboración propia con información de la empresa (2020)

Debido a que el área de confección no cumple con la meta de producción y se queda muy por debajo, afecta el cumplimiento de las áreas siguientes. Esto es ocasionado por las reclamaciones por parte de calidad por problemas que se encuentran en la prenda, ocasionando que se realicen retrabajos en confección y sea el área de acabado quien realice esa inspección en el retrabajo, para certificar que este se lleve bien y no exista una pérdida de producto. El porcentaje promedio de reclamación de producto es del $20 \%$ respecto a la meta a alcanzar en el área de confección. 
En el área de confección la experiencia del operador es muy importante, debido a que se realizan diferentes operaciones complejas, entre ellas, el corte del cuerpo (son los lienzos completos de tela del área de tejido), la unión de los cortes, el rétame que es coser la sobre costura de la prenda para que no sean visibles los hilos sobrantes y por último el deshebrado de la prenda. Las máquinas que se emplean para llevar a cabo las operaciones anteriores, a excepción del deshebrado que es totalmente manual, se muestran en la tabla 1, junto con el porcentaje de conocimiento que cada operador tiene en el manejo de cada máquina, de acuerdo con la opinión de los mismos trabajadores. La X significa que no tienen conocimiento.

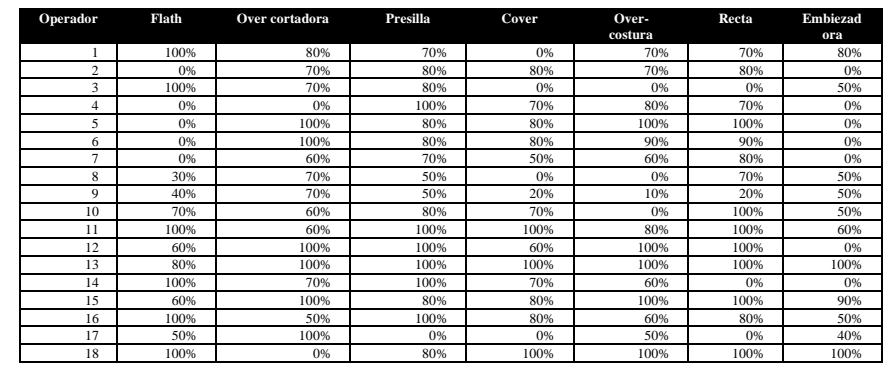

Tabla 1 Porcentaje de conocimiento en máquinas del área de confección

Fuente: Elaboración propia con información de la empresa (2020)

Esto no quiere decir que realmente conozcan el porcentaje del manejo de las máquinas; este es un diagnóstico dado por los operadores, ya que así lo consideran, lo cual vuelve a la evaluación del desempeño subjetiva. Es necesario evaluar objetivamente cuáles operaciones pueden hacer en estas máquinas, porque es ahí donde surge el problema, pues sabrán operarlas de manera general pero no tienen el conocimiento sobre qué operaciones realizar o cómo hacerlas.

\section{Metodología}

La metodología que se utilizó en el desarrollo de esta investigación está estructurada por las siguientes etapas:

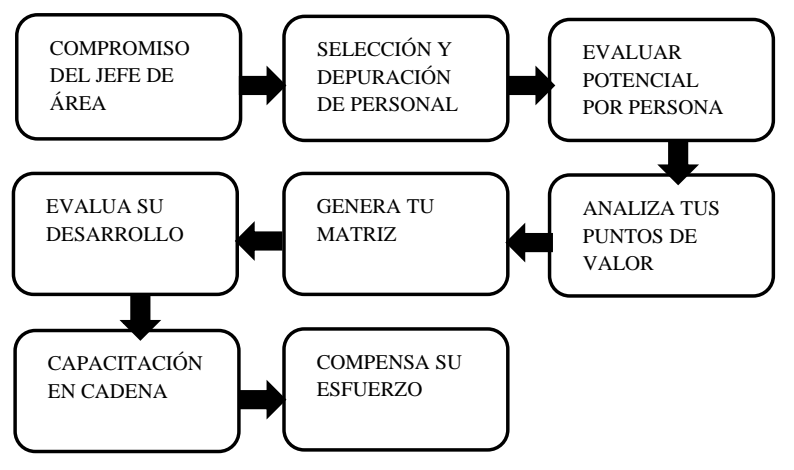

Figura 1 Porcentaje de conocimiento en máquinas del área de confección

Fuente: Elaboración propia con información de la empresa (2020)

\section{Compromiso del jefe de área}

Para comenzar, el primer paso es obtener el compromiso del jefe de área. Es necesario tener el compromiso del jefe, ya que, si éste se resiste al cambio que se generará en su área, nunca podrá darse cuenta de que puede generar un mayor resultado productivo en su área beneficiando a todos de manera positiva. El jefe de área firmó un documento de carácter tipo informativo donde da a conocer al departamento de recursos humanos que esta consiente de la generación de la matriz de habilidades y apoyar en lo que sea necesario a la realización de la mismo y de la estandarización de su método de trabajo para que este sea reflejado dentro de su productividad y eficiencia.

\section{Selección y depuración del personal}

Dentro de este punto se realizó con la ayuda del jefe una depuración del personal, esto quiere decir saber quién si estará comprometido con la idea de la nueva implementación y quienes no, así mismo sabremos realmente que persona le agregara un valor importante al proceso ya que ningún operador fue obligado a la realización de este proyecto, solo aquellos que son los comprometidos serán tomados en cuenta, dado el caso de no aceptar serán movidos a otras operaciones. El operador 3, fue el que no acepto.

\section{Evaluar el potencial personal}

En este punto es considerado conocer más allá de las habilidades y estudios con los que cuentan los operadores, el hecho de una buena elaboración de una matriz es conocer otros aspectos de ellos, como sus gustos o lo que les apasiona, esto puede no tener sentido pero tiene una gran importancia dentro de este proceso. 
El conocer bien a cada uno ayuda a que sea más fácil el proceso de adaptación, también es necesario evaluar hasta qué grado de estudios son con los que cuentan y así determinar más su valor dentro del área, pues una persona con mayor conocimiento tiene el potencial de escalar puestos más arriba de un operador (ver Tabla 2). Esta información se obtuvo preguntando a cada operador del área de confección.

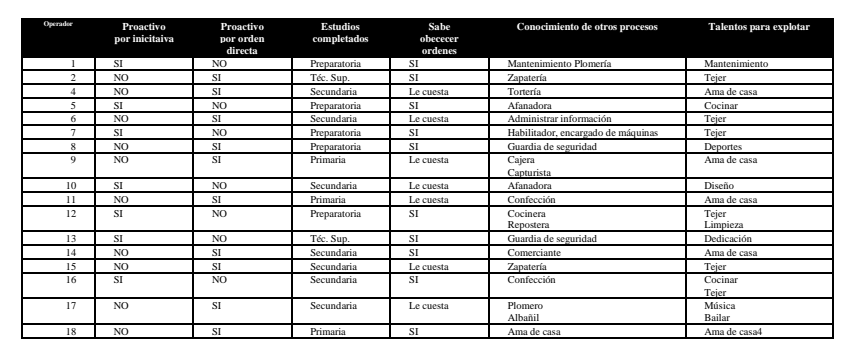

Tabla 2 Evaluación del potencial de los operadores del área de confección

Fuente: Elaboración propia con información de la empresa (2020)

Además de los aspectos considerados en la tabla, se integraron tanto la madurez sobre el trabajo como la salud física. Para estos aspectos, se determinó que todos los operadores cuentan con ellos.

\section{Analizar los puntos de valor}

Dentro de este punto se consideran las operaciones que causan un mayor impacto en el área, para que, en caso de ausentismo, no se atrase la producción y se pueda suplir sin riesgo de tener problemas de calidad, por falta de capacitación de quien sustituya. No es la clave capacitar en su mayoría en todas las operaciones, solo será en aquellas que son relevantes en el área y así consecutivamente una que otra hasta que la matriz de habilidades muestre que la mayoría del personal está capacitado.

Hay casos especiales en que la operación requiera de estudios o una preparación previa, es por lo que al saber estos puntos de valor del área de confección se dará una mayor prioridad para dedicación en el aprendizaje de los operadores y así mismo no se necesite de un capacitador externo. En la tabla 3 se muestran los puntos de valor en el área de confección para las actividades específicas. Estos valores fueron asignados en conjunto con el responsable del área.

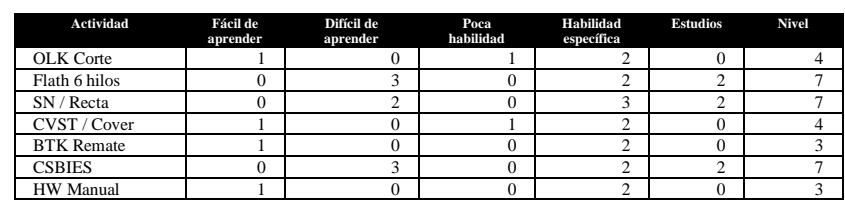

Tabla 3 Puntos de valor de las operaciones del área de confección

Fuente: Elaboración propia con información de la empresa (2020)

Es necesario mencionar que no todas las operaciones de la tabla, las llevan a cabo en todas las prendas, esto depende del tipo de prenda que se confeccione. Las operaciones que tengan un puntaje de 7 a 9 son las principales operaciones que afectarían el proceso de confección en caso de una ausencia, es por lo que, se comenzara a trabajar en flath, SN Recta y CVST Bies, seguidas de OLK Over y CVST Sobrecoser y por último HW Manual y BTK Remate, que son operaciones más fáciles porque cualquier persona puede realizarlas sin una preparación previa.

\section{Genera tu matriz}

En este punto se construye la matriz de habilidades. Para ello es necesario considerar que máquinas saben operar los trabajadores, considerando que ya saben realizar todas las operaciones; esta información se validó con un estudio de tiempos y movimientos. En la tabla 4 se muestra lo que sería la matriz inicial en maquinaria y así mismo partiremos para la realización de un plan de capacitación de como seria impartido y dando prioridad a los puntos de valor.

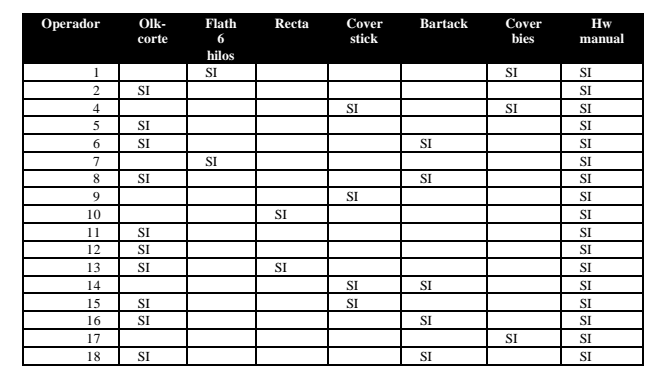

Tabla 4 Tabla de multi habilidades para los operadores del área de confección Fuente: Elaboración propia con información de la empresa (2020) 
Los operadores ya cuentan con algunos conocimientos previos de la operación de sus máquinas, pero no en su mayoría, es por esta razón que al ausentarse un operador surgen problemas para saber quién será el operador que ocupe la máquina para realizar dicha operación Un ejemplo presente es cuando surge la ausencia de un operador que opera flath, al solo existir dos operadores con conocimientos de esta máquina, hay un grave problema por el estilo que al trabajar necesite de esta máquina y dicha operación, lo que provoca baja eficiencia en cuestión de trabajo por día, provocando el incumplimiento de la meta diaria en el área de confección: ya que con solo dos operadores con conocimientos en flath no da abasto a toda el área en cuestión de cambio de estilos que requieran operaciones de esta máquina, es por ello que se decide realizar un plan de capacitación constante para el desarrollo de los operadores en la adquisición de más conocimiento en más máquinas y operaciones, ayudando a reducir el problema de producción.

A partir de este paso es posible establecer el esquema en el cual se dará la capacitación, si es necesario días completos, por horas, en máquinas, en software, capacitación externa o interna. Esto permitirá tener un control de lo que será la capacitación en cadena y se tenga la correcta supervisión.

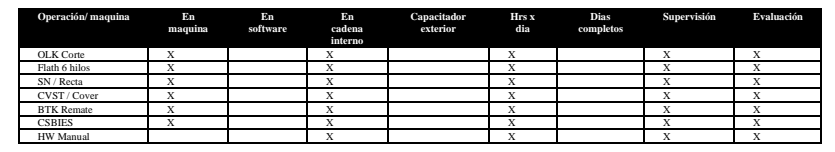

Tabla 5 Tabla de capacitación multi habilidades para operadores del área de confección

Fuente: Elaboración propia con información de la empresa (2020)

De acuerdo con la tabla 5, se establece que toda la capacitación será en máquina, impartida internamente por los mismos operadores y serán utilizadas horas/ días para que no se afecte de manera importante la jornada laboral, al final se aplica una evaluación de conocimiento para que sean capaces de enseñar a otros. El jefe de área de confección será quien monitoree en su mayoría el proceso ya que conoce el $100 \%$ del manejo de las máquinas, esto evitará problemas en la capacitación en cadena.

\section{Evalúa su desarrollo}

En esta etapa se estableció el método óptimo para trabajar en las máquinas del área de confección. Para ello se llevó a cabo un estudio de micro movimientos, para cada operador, en diferentes operaciones, con el uso de diagramas bimanuales. Se consideraron diferentes tipos de prendas de temporada; y se evalúo la calidad de la prenda al final de cada operación. La tabla 6 muestra el resumen de los estudios derivados de la presente investigación.

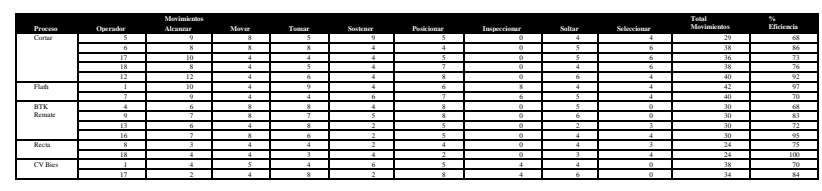

Tabla 6 Resumen de estudio de micro movimientos de operaciones en el área de confección

Fuente: Elaboración propia con información de la empresa (2020)

De la tabla 6 se determina que el operador 12 realiza mayor número de movimientos, pero de igual manera es uno de los que más defectos identifica y que al mismo tiempo es quien menos retrabajos hace, así que por parte de corte será quien capacite en su máquina ya que obtiene el $92 \%$ de efectividad. En el caso de Flath será el operador 1 quien capacitara, al haber obteniendo un $97 \%$ de efectividad, posteriormente para capacitar en BTK será el operador 16 ya que cuenta con una efectividad del 95\%; el operador 18 será quien de la capacitación sobre su máquina ya que por la experiencia que tiene cumple con el $100 \%$ de efectividad y por último será el operador 17 quien imparta la capacitación sobre la CS bies. Cuando exista la ausencia de alguien en la matriz se buscará quien es el más capaz para suplir el lugar de dicho operador y así mismo no existan problemas en producción.

\section{Capacitación en cadena}

En este punto se dará la capacitación. Cada operador se le será asignado operaciones de las cuales deberá cumplir durante un mes, para que posteriormente sean evaluados y se pueda liberar o seguir en capacitación hasta que sea apto de ser liberado al cumplir del 80-85\% de eficiencia (ver tabla 7). 


\begin{tabular}{|l|r|r|r|r|r|r|}
\hline $\begin{array}{c}\text { Operación / } \\
\text { máquina }\end{array}$ & Capacitador & \multicolumn{7}{c|}{ Operador } \\
\hline OLK Cortar & 12 & 1 & 4 & 7 & 9 & 10 \\
\hline Flath & 1 & 4 & 5 & 8 & 12 & 10 \\
\hline SN Recta & 10 & 1 & 2 & 5 & 6 & 11 \\
\hline BTK Remate & 16 & 5 & 9 & 10 & 17 & \\
\hline CS Bies & 17 & 2 & 6 & 15 & 10 & \\
\hline
\end{tabular}

Tabla 7 Plan de capacitación en cadena para el área de confección

Fuente: Elaboración propia con información de la empresa, (2020)

Una vez realizada la capacitación, se volvieron a medir las eficiencias, las cuales se reflejan en la tabla 8.

\begin{tabular}{|c|c|c|c|c|c|c|}
\hline Operación & Capacitador & \multicolumn{5}{|c|}{ Operador } \\
\hline \multirow{2}{*}{$\begin{array}{l}\text { OLK } \\
\text { Cortar }\end{array}$} & \multirow[t]{2}{*}{12} & 1 & 4 & 7 & 9 & 10 \\
\hline & & $88 \%$ & $75 \%$ & $94 \%$ & $95 \%$ & $80 \%$ \\
\hline \multirow[t]{2}{*}{ Flath } & \multirow[t]{2}{*}{1} & 4 & 5 & 8 & 12 & 10 \\
\hline & & $83 \%$ & $87 \%$ & $85 \%$ & $93 \%$ & $77 \%$ \\
\hline \multirow[t]{2}{*}{ SN Recta } & \multirow[t]{2}{*}{10} & 1 & 2 & 5 & 6 & 11 \\
\hline & & $80 \%$ & $75 \%$ & $88 \%$ & $87 \%$ & $80 \%$ \\
\hline \multirow{2}{*}{$\begin{array}{l}\text { BTK } \\
\text { Remate }\end{array}$} & \multirow[t]{2}{*}{16} & 5 & 9 & 10 & 17 & \\
\hline & & $90 \%$ & $78 \%$ & $80 \%$ & $86 \%$ & \\
\hline \multirow[t]{2}{*}{ CS Bies } & \multirow[t]{2}{*}{17} & 2 & 6 & 15 & 10 & \\
\hline & & $86 \%$ & $98 \%$ & $83 \%$ & $85 \%$ & \\
\hline
\end{tabular}

Tabla 8 Porcentaje de eficiencia alcanzada después de la capacitación en cadena, en el área de confección

Fuente: Elaboración propia con información de la empresa (2020)

\section{Compensa tu esfuerzo}

En este último punto el departamento de Recursos Humanos será el que tenga el trabajo de gratificar el esfuerzo de los operadores. Los resultados de las matrices deberán estar reflejados en la productividad del área y al mismo tiempo RRHH deberá estar al pendiente del esfuerzo de los operadores para su reconocimiento sobre el esfuerzo de ellos.

Para aquellos operadores que son ya capaces de enseñar a otros, se les otorgará un bono extra mensual y para aquellos que cumplen o cumplieron con más del $80 \%$ de las habilidades serán recompensados por vales de prendas mensuales y un pequeño bono mensual. Esta es una manera de motivar a todo empleado a ser multifuncional, pero al mismo tiempo la empresa ahorra costos de retrabajos y prendas de segunda que algunas veces no se suelen ya vender.

\section{Resultados}

Gracias a la capacitación sobre un método validado se logró reducir el $60 \%$ de los retrabajos en el área de confección por problemas de calidad. Se logró así mismo aumentar la productividad del área y de las áreas subsiguientes. Mejorando además la motivación de todos los trabajadores involucrados en el proyecto.

\section{Agradecimiento}

Agradecemos a Enrique Juárez de la Rosa por su invaluable apoyo, a la empresa caso de estudio y al Instituto Tecnológico Superior de San Martín Texmelucan, la financiación de la publicación del presente artículo.

\section{Conclusiones}

La implementación de mejoras en el proceso influye directamente en la calidad ofrecida y en el aumento de la producción, el proyecto llevado a cabo en la empresa, ha permitido el aumento de producción en el área de confección, por ende, la disminución de días de atraso de dicha área, por otra parte, con la estandarización del proceso se redujeron las reclamaciones por los defectos. Es de gran importancia para las empresas tener establecido un método que permita obtener un mejor control, orden y estandarización en los procesos.

Implantar herramientas ingenieriles en procesos productivos ayuda cumplir con la eficiencia y productividad y así mismo afectar de manera positiva la calidad requerida por los clientes aumentado su satisfacción, a su vez ayuda a lograr un mejor desempeño de los empleados para en un futuro alcanzar a certificarse bajo alguna norma de calidad.

Con base a los resultados se puede concluir que con la estandarización del proceso y capacitación en cadena se logró reducir los tiempos de producción logrando así mayor productividad, mayor cumplimiento de la demanda, producto con mayor calidad, disminución de pérdidas por retrabajos y piezas de segunda calidad. 


\section{Sugerencias de la investigación}

Se recomienda implementar planes de capacitación para todas las áreas, para un entrenamiento y conocimiento sobre la metodología a desempeñar en el proceso de cada referencia y procedimiento a seguir para que de esta manera se pueda alcanzar un ritmo de trabajo adecuado, no se tengan reprocesos y se puedan lograr los objetivos trazados por la empresa. Para personal nuevo es necesario impartir una capacitación adecuada para la mejor comprensión del proceso, y evitar la rotación de personal, por la capacitación ineficiente dada.

Por políticas de confidencialidad, el nombre de la empresa se mantiene privado.

\section{Referencias}

Bazan, A. (2019). Mejora de métodos de trabajo en el proceso de conserva de alcachofa para aumentar la productividad en una empresa agroindustrial en el año 2018. Universidad Nacional de Trujillo ; Repositorio institucional UNITRU. Recuperado de: http://dspace.unitru.edu.pe/handle/UNITRU/13 435; el 25 de Abril de 2020.

Cabanillas, J. y León Ríos, J. (2020). Aplicación de las herramientas de la ingeniería de métodos en el cultivo de arroz para incrementar la rentabilidad en las empresas agrícolas del Valle Jequetepeque. Universidad Nacional de Trujillo ; Repositorio institucional - UNITRU. Recuperado de: http://dspace.unitru.edu.pe/handle/UNITRU/15 460; el 27 de Abril de 2020.

Castillo, O. (2019). Gestión de Mantenimiento en la mejora de métodos de trabajo para disminuir los costos de mantenimiento preventivo en una empresa cementera. Universidad Nacional de Trujillo ; Repositorio institucional - UNITRU. Recuperado de: http://dspace.unitru.edu.pe/handle/UNITRU/12 678; el 26 de Abril de 2020.

CEMDA. (2019). Promoción de la economía circular en el sector moda y textil en México. Recuperado de: https://www.cemda.org.mx/wpcontent/uploads/2019/08/CEM_moda_publicaci ón.pdf; el 18 de Abril de 2020.
Gobierno Fácil. (2020). Plataforma de la Industria de la Moda ProMéxico. Recuperado de:

http://gobiernofacil.com/proyectos/plataformapromexico; el 19 de Abril de 2020.

Janania Abraham, C. (2008). Manual de tiempos y movimientos (Ed. Rev.). México, México: Limusa.

Kanawaty, G. (1998). Introducción al estudio de trabajo ( $4^{\mathrm{a}}$ ed.). Ginebra, Suiza: Oficina Internacional del Trabajo Ginebra.

Krick, E. V. (1977). Ingeniería de métodos ( $1^{\mathrm{a}}$ ed.). Limusa, México.

Lowry, S. M., H. B. Maynard y G. J. Stegemerten. (1940) Time and Motion Study and Formulas for Wage Incentive, (3a. Ed.) Nueva York: McGraw-Hill.

Niebel, W., \& Freivalds, A. (2009). Ingeniería industrial: Métodos, estándares y diseño del trabajo. (12 ed.). D.F, México: Mc Graw Hill.

Ramírez, J. (2015). Mejora del proceso de fabricación de bastidores estructurales mediante celda de soldadura robotizada en empresa metal mecánica. Universidad Nacional de Trujillo ; Repositorio institucional UNITRU. Recuperado de: http://dspace.unitru.edu.pe/handle/UNITRU/22 27; el 23 de Abril de 2020.

Ruiz, H. (2016). Estudio de métodos de trabajo en el proceso de llenado de tolva para mejorar la productividad de la empresa agrosemillas don benjamín e.i.r.l. Universidad Nacional de Trujillo ; Repositorio institucional - UNITRU. Recuperado de: http://dspace.unitru.edu.pe/handle/UNITRU/11 03; el 24de Abril de 2020.

Secretaría de Economía. (2019). Informe 2017 2018. Delegación Federal de la Secretaría de Economía del Estado de Puebla. Coordinación General de Delegaciones Federales. Recuperado de:

https://www.gob.mx/cms/uploads/attachment/fi le/384792/21_Puebla_Informe_de_Labores_20 17-2018_002_.pdf; el 17 de Abril de 2020. 